\title{
TRIBONACCI DIOPHANTINE QUADRUPLES
}

\author{
Carlos Alexis Gómez Ruiz and Florian Luca \\ Universidad del Valle, Colombia and University of the Witwatersrand, South \\ Africa
}

\begin{abstract}
In this paper, we show that there does not exist a quadruple of positive integers $a_{1}<a_{2}<a_{3}<a_{4}$ such that $a_{i} a_{j}+1(i \neq j)$ are all members of the Tribonacci sequence $\left(T_{n}\right)_{n \geq 0}$.
\end{abstract}

\section{INTRODUCTION}

A Diophantine $m$-tuple is a set $a_{1}, \ldots, a_{m}$ of positive rational numbers, or integers, such that $a_{i} a_{j}+1$ is a square for all $1 \leq i<j \leq m$. The problem of finding Diophantine $m$-tuples has a long history. Diophantus found the rational quadruple $\{1 / 16,33 / 16,17 / 4,105 / 16\}$ and only long after Fermat found the integer quadruple $\{1,3,8,120\}$. In the integer case, it is known that there are infinitely many Diophantine quadruples of integers. Recently, there has been remarkable progress by Dujella $([5])$, who showed that there is no Diophantine sextuple and that there can be at most finitely many Diophantine quintuples and all of them are, at least in theory, effectively computable. However, in the rational case, it is not even known if $m$ must be universally bounded. Gibbs ([7]), found a few examples with $m=6$.

Natural generalizations of this problem have been studied by replacing the squares by higher powers of fixed, or variable exponents in $[1,2,8-10]$, or by members of nondegenerate binary recurrences $\left(u_{n}\right)_{n \geq 0}$, by Fuchs, Luca and Szalay in [6]. In this last setting, some research was done concerning Diophantine triples $(a, b, c)$ of distinct positive integers, such that $a b+1, a c+1$ and $b c+1$ are all three members of a particular recurrence $\left(u_{n}\right)_{n \geq 0}$. Particularly, Luca and Szalay showed that there are no Diophantine triples with values in the Fibonacci sequence (see [11]) and that the only Diophantine

2010 Mathematics Subject Classification. 11B37, 11B39, 11D61.

Key words and phrases. Quadruples Diophantine, Tribonacci numbers. 
triple with values in the Lucas companion $\left(L_{n}\right)_{n \geq 0}$ of the Fibonacci sequence is $(a, b, c)=(1,2,3)$ (see [12]).

In this paper, we look at Diophantine quadruples with value in the Tribonacci sequence. The Tribonacci sequence $\left(T_{n}\right)_{n \geq 0}$ starts with $T_{0}=0$ and $T_{1}=T_{2}=1$ and each term afterwards is the sum of the preceding three terms

$$
T_{n+3}=T_{n+2}+T_{n+1}+T_{n}, \quad \text { for all } \quad n \geq 0 .
$$

We prove the following theorem.

MAIN TheOREM. There do not exist positive integers $a_{1}<a_{2}<a_{3}<a_{4}$ such that $a_{i} a_{j}+1=T_{n_{i, j}}$, with $1 \leq i<j \leq 4$, for some integers positive $n_{i, j}$.

We conjecture that in fact there are only finitely many Tribonacci tripes $(a, b, c)$, that is triples of positive integers $a<b<c$ such that $a b+1, a c+$ $1, b c+1$ are all three Tribonacci numbers, but we do not know how to attack this question. We leave this as an open question for the reader.

\section{Properties}

The characteristic polynomial of the Tribonacci sequence is

$$
\Psi(x)=x^{3}-x^{2}-x-1 .
$$

It has a real root

$$
\alpha=\frac{1}{3}\left(1+(19-3 \sqrt{33})^{1 / 3}+(19+3 \sqrt{33})^{1 / 3}\right)
$$

and two complex conjugated roots

$$
\beta=\alpha^{-1 / 2} e^{i \theta} \text { and } \gamma=\alpha^{-1 / 2} e^{-i \theta} \text { with } \theta \in(\pi / 2, \pi) .
$$

Using a result of Dresden ([4]), we have the following Binet-like formula for $T_{n}$ :

$$
T_{n}=c_{\alpha} \alpha^{n-1}+c_{\beta} \beta^{n-1}+c_{\gamma} \gamma^{n-1},
$$

where $c_{z}=(z-1) /(4 z-6)$. Dresden also showed that the contribution of the complex roots $\beta$ and $\gamma$, which have absolute value less that 1 , to the right-hand side of (2.2) is very small. More precisely, he proved that the inequality

$$
\left|T_{n}-c_{\alpha} \alpha^{n-1}\right|<\frac{1}{2} \quad \text { holds for all } \quad n \geq 0 .
$$

Another well-known property of the Tribonacci numbers which is useful to us is the following (see [3]):

$$
\alpha^{n-2} \leq T_{n} \leq \alpha^{n-1} \quad \text { for all } \quad n \geq 1 .
$$




\section{First OBSERVATIONS}

Let $a_{1}<a_{2}<a_{3}<a_{4}$ be a Tribonacci Diophantine quadruple. Then

$$
a_{1} a_{2}+1=T_{x}, a_{2} a_{3}+1=T_{y}, a_{3} a_{4}+1=T_{z}, a_{1} a_{4}+1=T_{w},
$$

for some positive integers $x, y, z$ and $w$. We see easily that

$$
4 \leq x<\min \{y, w\} \leq \max \{y, w\}<z .
$$

Combining the equalities in (3.1), we conclude that

$$
\left(T_{x}-1\right)\left(T_{z}-1\right)=\left(T_{y}-1\right)\left(T_{w}-1\right) .
$$

From inequalities (2.4) we have $\alpha^{n-2.5}<T_{n}-1<\alpha^{n-1}$, for all $n \geq 4$. Thus,

$$
\alpha^{x+z-5}<\left(T_{x}-1\right)\left(T_{z}-1\right)<\alpha^{x+z-2}
$$

and

$$
\alpha^{y+w-5}<\left(T_{y}-1\right)\left(T_{w}-1\right)<\alpha^{y+w-2} .
$$

Hence, considering equality (3.3), we deduce that

$$
|(x+z)-(y+w)| \leq 2 .
$$

In the rest of this paper, we work on the Diophantine equation (3.3) by distinguishing two cases:

$$
x+z \neq y+w \text { and } x+z=y+w .
$$

4. The CASE $x+z \neq y+w$

By using formula (2.2) and inequality (2.3), we have that

$$
T_{n}=c_{\alpha} \alpha^{n-1}+e(n), \text { with }|e(n)|<1 / 2 .
$$

Thus, by expanding equation (3.3) this can be rewritten as

$$
\begin{aligned}
c_{\alpha}^{2} \alpha^{x+z-2}-c_{\alpha}^{2} \alpha^{y+w-2}= & c_{\alpha}(1-e(z)) \alpha^{x-1}+c_{\alpha}(1-e(x)) \alpha^{z-1} \\
& +c_{\alpha}(e(w)-1) \alpha^{y-1}+c_{\alpha}(e(y)-1) \alpha^{w-1}+e(x) \\
& +e(z)-e(y)-e(w)-e(x) e(z)+e(y) e(w) .
\end{aligned}
$$

Dividing both sides of the above equation by $c_{\alpha}^{2} \alpha^{x+z-2}$ and taking absolute values, we get

$$
\begin{aligned}
\left|1-\alpha^{-(x+z-y-w)}\right| & <\frac{3}{2 c_{\alpha}}\left(\frac{1}{\alpha^{z-1}}+\frac{1}{\alpha^{x-1}}+\frac{\alpha^{y-z}}{\alpha^{x-1}}+\frac{\alpha^{w-z}}{\alpha^{x-1}}\right)+\frac{2.5}{c_{\alpha}^{2} \alpha^{x+z-2}} \\
(4.2) & <\frac{1}{\alpha^{x-1}}\left(\frac{3}{c_{\alpha}}\left(1+\frac{1}{\alpha}\right)+\frac{5}{2 c_{\alpha}^{2} \alpha^{5}}\right)<\frac{8}{\alpha^{x-1}},
\end{aligned}
$$

where we have used (3.2) and that $|e(n)-1|<3 / 2$. On the other hand, by inequality (3.4) and since $x+z \neq y+w$, we obtain

$$
\min _{|x+z-y-w| \leq 2}\left|1-\alpha^{-(x+z-y-w)}\right|>0.4563 .
$$


Thus, by (3.2), (4.2) and (4.3), we get $x=4$ or 5 .

Now, from equation (3.3) we have the equality

$$
\left(T_{x}-1\right) T_{z}-T_{\lambda} T_{\delta}=T_{x}-T_{\lambda}-T_{\delta},
$$

where $\lambda=\min \{y, w\} \leq \delta=\max \{y, w\}$. Replacing $T_{z}, T_{\lambda}, T_{\delta}$ according to the equation (4.1) in the above equation, we conclude that

$$
\begin{aligned}
\left(T_{x}-1\right) c_{\alpha} \alpha^{z-1}-c_{\alpha}^{2} \alpha^{\lambda+\delta-2}= & c_{\alpha}(e(\delta)-1) \alpha^{\lambda-1}+c_{\alpha}(e(\lambda)-1) \alpha^{\delta-1}-e(\lambda) \\
& -e(\delta)+e(\lambda) e(\delta)-\left(T_{x}-1\right)(e(z)-1)+1 .
\end{aligned}
$$

Dividing both sides of above equation by $\left(T_{x}-1\right) c_{\alpha} \alpha^{z-1}$, and taking absolute values, we get

$$
\left|1-\left(T_{x}-1\right)^{-1} c_{\alpha} \alpha^{\lambda+\delta-z-1}\right|<\frac{1 / 2}{\alpha^{z-\lambda}}+\frac{1 / 2}{\alpha^{z-\delta}}+\frac{9 /\left(4 c_{\alpha} \alpha^{4}\right)}{\alpha^{z-5}}<\frac{1.4}{\alpha^{z-\delta}},
$$

where we used the fact that $z-5 \geq z-\lambda \geq z-\delta$ (by (3.2)) and that $T_{x}-1 \geq 3$. However, by inequality (3.4) and since $x=4$ or 5 , we obtain that $|\lambda+\delta-z-1| \leq 6$. We find that

$$
\min _{\substack{x \in\{4,5\} \\|\lambda+\delta-z-1| \leq 6}}\left|1-\left(T_{x}-1\right)^{-1} c_{\alpha} \alpha^{\lambda+\delta-z-1}\right|>0.179 .
$$

Thus, combining (3.2), (4.4) and (4.5) we conclude that $z-\delta=1,2$ or 3 . Returning to inequality (3.4), we get that $5 \leq \lambda \leq 10$.

Going back one more time to equality (3.3), we rewrite it as

$$
\left(T_{x}-1\right) T_{z}-\left(T_{\lambda}-1\right) T_{\delta}=T_{x}-T_{\lambda} .
$$

Replacing $T_{z}, T_{\delta}$ according to (4.1), dividing by $\left(T_{\lambda}-1\right) c_{\alpha} \alpha^{\delta-1}$ and taking absolute values, we get

$$
\left|1-\left(T_{x}-1\right)\left(T_{\lambda}-1\right)^{-1} \alpha^{z-\delta}\right|<\frac{5}{\alpha^{\delta-1}} .
$$

By analyzing the minimum value of the left hand side in (4.6), we get

$$
\min _{\substack{x \in\{4,5\} \\ 5 \leq \lambda \leq 10 \\ z-\delta \in\{1,2,3\}}}\left|1-\left(T_{x}-1\right)\left(T_{\lambda}-1\right)^{-1} \alpha^{z-\delta}\right|>0.08 .
$$

Hence, from inequalities (4.6) and (4.7) we conclude that $\delta \leq 7$ and, in particular, that $6 \leq z \leq 10$.

Let us record what we have proved so far.

LEMMA 4.1. Let $4 \leq x<\min \{y, w\} \leq \max \{y, w\}<z$ be positive integers such that

$$
x+z \neq y+w \quad \text { and } \quad\left(T_{x}-1\right)\left(T_{z}-1\right)=\left(T_{y}-1\right)\left(T_{w}-1\right) .
$$

Then

$$
4 \leq x \leq 5, \quad 5 \leq y, w \leq 7 \quad \text { and } \quad 6 \leq z \leq 10
$$




\section{The CASE $x+z=y+w$}

Substituting $\beta$ and $\gamma$ given by (2.1) in equation (2.2), we conclude that

$$
T_{n}-1=c_{\alpha} \alpha^{n-1}+\frac{\widetilde{c}(n)}{\alpha^{\frac{1}{2}(n-1)}}-1=c_{\alpha} \alpha^{n-1}\left(1+\frac{\widetilde{c}(n) / c_{\alpha}}{\alpha^{\frac{3}{2}(n-1)}}-\frac{1 / c_{\alpha}}{\alpha^{n-1}}\right),
$$

where $\widetilde{c}(n):=c_{\beta} e^{i \theta(n-1)}+c_{\gamma} e^{-i \theta(n-1)}$.

Combining the above equation with (3.3) and performing the respective calculations, we get an equality with dominant terms $1 / \alpha^{\lambda-1}$ and $1 / \alpha^{x-1}$ in each of the two sides. Grouping these terms in one side, we obtain

$$
\begin{aligned}
\frac{1 / c_{\alpha}}{\alpha^{\lambda-1}}-\frac{1 / c_{\alpha}}{\alpha^{x-1}}= & \frac{\widetilde{c}(\delta) / c_{\alpha}}{\alpha^{\frac{3}{2}(\delta-1)}}+\frac{\widetilde{c}(\lambda) / c_{\alpha}}{\alpha^{\frac{3}{2}(\lambda-1)}}-\frac{\widetilde{c}(z) / c_{\alpha}}{\alpha^{\frac{3}{2}(z-1)}}-\frac{\widetilde{c}(x) / c_{\alpha}}{\alpha^{\frac{3}{2}(x-1)}}+\frac{1 / c_{\alpha}}{\alpha^{z-1}} \\
& -\frac{1 / c_{\alpha}}{\alpha^{\delta-1}}-\frac{\widetilde{c}(\lambda) / c_{\alpha}^{2}}{\alpha^{\frac{3}{2}(\lambda-1)+\delta-1}}-\frac{\widetilde{c}(\delta) / c_{\alpha}^{2}}{\alpha^{\frac{3}{2}(\delta-1)+\lambda-1}} \\
& +\frac{\widetilde{c}(x) / c_{\alpha}^{2}}{\alpha^{\frac{3}{2}(x-1)+z-1}}+\frac{\widetilde{c}(z) / c_{\alpha}^{2}}{\alpha^{\frac{3}{2}(z-1)+x-1}}+\frac{(\widetilde{c}(\lambda) \widetilde{c}(\delta)-\widetilde{c}(x) \widetilde{c}(z)) / c_{\alpha}^{2}}{\alpha^{\frac{3}{2}(x+z-2)}} .
\end{aligned}
$$

Multiplying both sides of (5.2) by $c_{\alpha} \alpha^{x-1}$ and taking absolute values, we conclude that

$$
\begin{aligned}
\left|1-\alpha^{-(\lambda-x)}\right|< & \frac{|\widetilde{c}(\delta)| / \alpha^{2}}{\alpha^{\delta-x}}+\frac{|\widetilde{c}(\lambda)| / \alpha}{\alpha^{\frac{1}{2}(x-1)}}+\frac{|\widetilde{c}(z)| / \alpha^{5 / 2}}{\alpha^{z-x}}+\frac{|\widetilde{c}(x)|}{\alpha^{\frac{1}{2}(x-1)}} \\
& +\frac{1}{\alpha^{z-x}}+\frac{1}{\alpha^{\delta-x}}+\frac{|\widetilde{c}(\lambda)| /\left(c_{\alpha} \alpha^{6}\right)}{\alpha^{\delta-x}}+\frac{|\widetilde{c}(\delta)| /\left(c_{\alpha} \alpha^{6}\right)}{\alpha^{\delta-x}} \\
& +\frac{|\widetilde{c}(x)| /\left(c_{\alpha} \alpha^{5}\right)}{\alpha^{\frac{1}{2}(x-1)}}+\frac{|\widetilde{c}(z)| /\left(c_{\alpha} \alpha^{5}\right)}{\alpha^{\frac{1}{2}(x-1)}} \\
& +\frac{(|\widetilde{c}(\lambda)||\widetilde{c}(\lambda)|+|\widetilde{c}(\lambda)||\widetilde{c}(\lambda)|) /\left(c_{\alpha} \alpha^{15 / 2}\right)}{\alpha^{\frac{1}{2}(x-1)}} \\
< & \frac{2.1}{\alpha^{\delta-x}}+\frac{0.3}{\alpha^{\frac{x}{2}}} \leq \frac{2.4}{\alpha^{\ell}}
\end{aligned}
$$

where $\ell:=\min \{x / 2, \delta-x\}$. In the above inequality, we used the facts that $|\widetilde{c}(n)| \leq\left|c_{\beta}\right| / 2<0.1$, for all $n \geq 4$, as well as inequalities (3.2). Thus, noting also that $\left|1-\alpha^{-(\lambda-x)}\right| \geq 1-1 / \alpha>0.45$, we obtain from (5.3) that $\alpha^{\ell}<5.4$, which leads to $\ell<2.8$.

If $\ell=x / 2$, we then get that $x=4$ or 5 . Returning to equation (3.3) and replacing $T_{\lambda}, T_{\delta}$ and $T_{z}$ (from the first part of (5.1)), we get an equality with dominant terms $c_{\alpha}^{2} \alpha^{\lambda+\delta-2}$ and $\left(T_{x}-1\right) c_{\alpha} \alpha^{z-1}$. Separating them into one side and putting all the rest into the other side, we obtain

$$
\begin{aligned}
& \left(T_{x}-1\right) c_{\alpha} \alpha^{z-1}-c_{\alpha}^{2} \alpha^{\lambda+\delta-2}=\frac{\widetilde{c}(\delta) c_{\alpha} \alpha^{\lambda-1}}{\alpha^{\frac{1}{2}(\delta-1)}}+\frac{\widetilde{c}(\lambda) c_{\alpha} \alpha^{\delta-1}}{\alpha^{\frac{1}{2}(\lambda-1)}}+\frac{\widetilde{c}(\lambda) \widetilde{c}(\delta)}{\alpha^{\frac{1}{2}(\lambda+\delta-2)}} \\
& -\frac{\widetilde{c}(z)\left(T_{x}-1\right)}{\alpha^{\frac{1}{2}(z-1)}}-\frac{\widetilde{c}(\delta)}{\alpha^{\frac{1}{2}(\delta-1)}}-\frac{\widetilde{c}(\lambda)}{\alpha^{\frac{1}{2}(\lambda-1)}}-c_{\alpha} \alpha^{\lambda-1}-c_{\alpha} \alpha^{\delta-1}+T_{x} .
\end{aligned}
$$


As usual, we divide both sides of the above equality by $c_{\alpha}^{2} \alpha^{\lambda+\delta-2}$ and take absolute values to get:

$$
\begin{aligned}
& \left|\left(T_{x}-1\right) c_{\alpha}^{-1} \alpha^{z-(\lambda+\delta)+1}-1\right|<\frac{|\widetilde{c}(\delta)| / c_{\alpha}}{\alpha^{\frac{3}{2}(\delta-1)}}+\frac{|\widetilde{c}(\lambda)| / c_{\alpha}}{\alpha^{\frac{3}{2}(\lambda-1)}}+\frac{|\widetilde{c}(\lambda)||\widetilde{c}(\delta)| / c_{\alpha}^{2}}{\alpha^{\frac{3}{2}(\lambda+\delta-2)}} \\
& +\frac{\left(|\widetilde{c}(z)| / c_{\alpha}^{2}\right)\left(T_{x}-1\right)}{\alpha^{\frac{1}{2}(z-1)+\lambda+\delta-2}}+\frac{|\widetilde{c}(\delta)| / c_{\alpha}^{2}}{\alpha^{\frac{3}{2}(\delta-1)+\lambda-1}} \\
& +\frac{|\widetilde{c}(\lambda)| / c_{\alpha}^{2}}{\alpha^{\frac{3}{2}(\lambda-1)+\delta-1}}+\frac{1 / c_{\alpha}}{\alpha^{\delta-1}}+\frac{1 / c_{\alpha}}{\alpha^{\lambda-1}}+\frac{T_{x} / c_{\alpha}^{2}}{\alpha^{\lambda+\delta-2}} \\
& <\frac{1}{\alpha^{\lambda-1}}\left(\frac{\left|c_{\beta}\right|}{c_{\alpha} \alpha^{2}}+\frac{\left|c_{\beta}\right|^{2}}{4 c_{\alpha}^{2} \alpha^{8}}+\frac{3\left|c_{\beta}\right|}{c_{\alpha}^{2} \alpha^{13 / 2}}+\frac{\left|c_{\beta}\right|}{c_{\alpha}^{2} \alpha^{6}}\right. \\
& \left.+\frac{2}{c_{\alpha}}+\frac{7}{c_{\alpha}^{2} \alpha^{4}}\right)<\frac{5}{\alpha^{\lambda-1}}
\end{aligned}
$$

In the above chain of inequalities, we used inequality (3.2) in each term of the right side and the fact that $T_{x} \leq T_{5}=7$. Further, since $z-(\lambda+\delta)=-x$ and $x=4$, or 5 , we get that

$$
\min _{x \in\{4,5\}}\left|\left(T_{x}-1\right) c_{\alpha}^{-1} \alpha^{z-(\lambda+\delta)+1}-1\right|>0.15
$$

Therefore, it follows from (3.2) and (5.4) that $\lambda=5$ or 6 . Once more, we return to equation (3.3). Replacing $T_{z}, T_{\delta}$ according to (5.1), dividing by $\left(T_{\lambda}-1\right) c_{\alpha} \alpha^{\delta-1}$, and taking absolute values, we have

$$
\begin{aligned}
\left|1-\left(T_{x}-1\right)\left(T_{\lambda}-1\right)^{-1} \alpha^{z-\delta}\right| & <\frac{1}{\alpha^{\delta-1}}\left(\frac{\left|c_{\beta}\right|}{2 c_{\alpha} \alpha^{2}}+\frac{\left|c_{\beta}\right|}{2 c_{\alpha} \alpha^{5 / 2}}+\frac{2}{c_{\alpha}}\right) \\
& <\frac{3.4}{\alpha^{\delta-1}} .
\end{aligned}
$$

Now, we estimate the minimum value of the left hand side in (5.5), where $z-\delta=\lambda-x, x=4$ or 5 , and $\lambda=5$ or 6 , to get

$$
\min _{\substack{x \in\{4,5\} \\ \lambda \in\{5,6\}}}\left|1-\left(T_{x}-1\right)\left(T_{\lambda}-1\right)^{-1} \alpha^{\lambda-x}\right|>0.08 .
$$

Hence, from inequalities (5.5) and (5.6) we conclude that $\delta \leq 7$ which implies that $6 \leq z \leq 9$.

But, if $\ell=\delta-x$ then $\lambda \leq \delta \leq x+2$. In particular, $x+z=\lambda+\delta \leq 2 x+4$, and so $z \leq x+4$. To summarize, we proved the next result.

LEMMA 5.1. Let $4 \leq x<\min \{y, w\} \leq \max \{y, w\}<z$ be positive integers such that $x+z=y+w$ and $\left(T_{x}-1\right)\left(T_{z}-1\right)=\left(T_{y}-1\right)\left(T_{w}-1\right)$. Then

$$
4 \leq x \leq 5, \quad 5 \leq y, w \leq 7 \quad \text { and } \quad 6 \leq z \leq 9,
$$

or

$$
x+1 \leq y, w \leq x+2 \quad \text { and } \quad x+2 \leq z \leq x+4 .
$$




\section{The Proof of the Main Theorem}

6.1. Case $x+z \neq y+w$. Listing the values of $T_{x}, T_{y}, T_{z}, T_{w}$, with $x, y, z, w$ in the range given by Lemma 4.1, we note that (3.3) has no solutions. So, there is no Tribonacci Diophantine quadruple that satisfies (3.1) in this case.

6.2. Case $x+z=y+w$. From the first conclusion of Lemma 5.1, we obtain by simple verification that the only solution of $(3.3)$ is $(x, y, z, w)=(4,5,6,5)$. Hence, from (3.1) we get

$$
a_{1} a_{2}=3, \quad a_{2} a_{3}=6, \quad a_{3} a_{4}=12, \quad a_{1} a_{4}=6,
$$

which does not lead to an integer solution $\left(a_{1}, a_{2}, a_{3}, a_{4}\right)$. So, there is no Tribonacci Diophantine quadruple that satisfies (3.1) in this case.

From the second conclusion of Lemma 5.1, we can rewrite the equation $(3.3)$ as

$$
\left(T_{x}-1\right)\left(T_{x+i+j}-1\right)=\left(T_{x+i}-1\right)\left(T_{x+j}-1\right),
$$

where $1 \leq i \leq j \leq 2$. Using identity (5.1) in above equation, and by making the respective calculations and simplifications, we get

$$
\begin{aligned}
\frac{1 / c_{\alpha}}{\alpha^{x+j-1}} & +\frac{1 / c_{\alpha}}{\alpha^{x+i-1}}-\frac{1 / c_{\alpha}}{\alpha^{x+i+j-1}}-\frac{1 / c_{\alpha}}{\alpha^{x-1}} \\
= & \frac{\widetilde{c}(x+i) \widetilde{c}(x+j) / c_{\alpha}^{2}}{\alpha^{\frac{3}{2}(2 x+i+j-2)}}-\frac{\widetilde{c}(x) \widetilde{c}(x+i+j) / c_{\alpha}^{2}}{\alpha^{\frac{3}{2}(2 x+i+j-2)}}-\frac{\widetilde{c}(x+i+j) / c_{\alpha}}{\alpha^{\frac{3}{2}(x+i+j-1)}} \\
& -\frac{\widetilde{c}(x) / c_{\alpha}}{\alpha^{\frac{3}{2}(x-1)}}+\frac{\widetilde{c}(x) / c_{\alpha}^{2}}{\alpha^{\frac{3}{2}(x-1)+x+i+j-1}}+\frac{\widetilde{c}(x+i+j) / c_{\alpha}^{2}}{\alpha^{\frac{3}{2}(x+i+j-1)+x-1}}+\frac{\widetilde{c}(x+j) / c_{\alpha}}{\alpha^{\frac{3}{2}(x+j-1)}} \\
& +\frac{\widetilde{c}(x+i) / c_{\alpha}}{\alpha^{\frac{3}{2}(x+i-1)}}-\frac{\widetilde{c}(x+i) / c_{\alpha}^{2}}{\alpha^{\frac{3}{2}(x+i-1)+x+j-1}}-\frac{\widetilde{c}(x+j) / c_{\alpha}^{2}}{\alpha^{\frac{3}{2}(x+j-1)+x+i-1}} .
\end{aligned}
$$

Multiplying both sides of the last equation by $c_{\alpha} \alpha^{x}$, and taking absolute values, we conclude that

$$
\begin{aligned}
\mid \frac{1}{\alpha^{j-1}} & +\frac{1}{\alpha^{i-1}}-\frac{1}{\alpha^{i+j-1}}-\alpha \mid \\
< & \frac{\left|c_{\beta}\right|^{2} / 2 c_{\alpha}}{\alpha^{\frac{1}{2}(4 x+3 i+3 j-6)}}+\frac{\left|c_{\beta}\right|}{\alpha^{\frac{1}{2}(x+3 i+3 j-3)}}+\frac{\left|c_{\beta}\right|}{\alpha^{\frac{1}{2}(x-3)}}+\frac{\left|c_{\beta}\right| / c_{\alpha}}{\alpha^{\frac{3}{2}(x-1)+i+j-1}} \\
& +\frac{\left|c_{\beta}\right| / c_{\alpha}}{\alpha^{\frac{3}{2}(x+i+j-1)-1}}+\frac{\left|c_{\beta}\right|}{\alpha^{\frac{1}{2}(x+3 j-3)}}+\frac{\left|c_{\beta}\right|}{\alpha^{\frac{1}{2}(x+3 i-3)}}+\frac{\left|c_{\beta}\right| / c_{\alpha}}{\alpha^{\frac{3}{2}(x+i-1)+j-1}} \\
& +\frac{\left|c_{\beta}\right| / c_{\alpha}}{\alpha^{\frac{3}{2}(x+j-1)+i-1}} .
\end{aligned}
$$

It is easy to check that for $x \geq 6$ and $1 \leq i \leq j \leq 2$, the above inequality is impossible. Thus, $x=4$ or 5 , and from Lemma 5.1 we obtain values for $y, w$ and $z$ which were already considered, and which do not lead to positive integer solutions to equation (3.1). This completes the proof of the main theorem. 
ACKNOWLEDGEMENTS.

The authors thank the referee for comments which improved the quality of this paper. C. A. G. thanks to the Universidad del Valle for support during his Ph.D. studies.

\section{REFERENCES}

[1] Y. Bugeaud and A. Dujella, On a problem of Diophantus for higher powers, Math. Proc. Cambridge Philos. Soc. 135 (2003), 1-10.

[2] Y. Bugeaud and K. Gyarmati, On generalizations of a problem of Diophantus, Illinois J. Math. 48 (2004), 1105-1115.

[3] J. Bravo and F. Luca, Powers of two in generalized Fibonacci sequences, Rev. Colombiana Mat. 46 (2012), 67-79.

[4] G. P. Dresden and Z. Du, A simplified Binet formula for $k$-generalized Fibonacci numbers, J. Integer Sequences 17 (2014), Article 14.4.7.

[5] A. Dujella, There are only finitely many Diophantine quintuples, J. Reine Angew. Math. 566 (2004), 183-214.

[6] C. Fuchs, F. Luca and L. Szalay, Diophantine triples with values in binary recurrences, Ann. Sc. Norm. Super. Pisa Cl. Sc. (5), 7 (2008), 579-608.

[7] P. Gibbs, Some rational Diophantine sextuples, Glas. Mat. Ser. III 41(61) (2006), 195-203.

[8] K. Gyarmati, A. Sarkozy and C. L. Stewart, On shifted products which are powers, Mathematika 49 (2002), 227-230.

[9] K. Gyarmati and C. L. Stewart, On powers in shifted products, Glas. Mat. Ser. III 42(62) (2007), 273-279.

[10] F. Luca, On shifted products which are powers, Glas. Mat. Ser. III 40(60) (2005), $13-20$.

[11] F. Luca and 1. Szalay, Fibonacci Diophantine triples, Glas. Mat. Ser. III 43(63) (2008), 253-264.

[12] F. Luca and 1. Szalay, Lucas Diophantine triples, Integers 9 (2009), 441-457.

C. A. Gómez

Departamento de Matemáticas

Universidad del Valle

25360 Cali, Calle 13 No 100-00

Colombia

E-mail: carlos.a.gomez@correounivalle.edu.co

F. Luca

School of Mathematics

University of the Witwatersrand

P. O. Box Wits

South Africa

E-mail: florian.luca@wits.ac.za

Received: 16.2.2014.

Revised: 23.4.2014. 